Georgian Mathematical Journal

Volume 12 (2005), Number 1, 65-74

\title{
NECESSARY OPTIMALITY CONDITIONS FOR LIPSCHITZ MULTIOBJECTIVE OPTIMIZATION PROBLEMS
}

\author{
N. GADHI
}

\begin{abstract}
Optimality conditions are established in terms of LagrangeKuhn-Tucker multipliers for multiobjective optimization problems by a scalarization technique. Throughout this note, the data are assumed to be locally Lipschitz.
\end{abstract}

2000 Mathematics Subject Classification: Primary 90C29; Secondary 49K99.

Key words and phrases: Necessary optimality conditions, multiobjective optimization, scalarization, mathematical programming.

\section{INTRODUCTION}

In the last decade there has been an increasing interest in set-valued optimization $[3,5,6,10,16,17,18,19]$. General optimization problems with set-valued constraints or a set-valued function are closely related to problems in stochastic programming, fuzzy programming and optimal control. If the values of a given function vary in a specified region, this fact could be described by using a membership function in the theory of fuzzy sets or using information on the distribution of the function values. In this general setting probability distributions or membership functions are not needed because only sets are considered. Optimal control problems with differential inclusions belong to this class of setvalued optimization problems as well. Set-valued optimization seems to have the potential to become a bridge between different areas in optimization; it is a substantial extension of the standard optimization theory.

In this note, we are concerned with the set-valued optimization problem

$$
(P):\left\{\begin{array}{c}
\min F(x), \\
\text { subject to } G(x) \cap-Z^{+} \neq \varnothing,
\end{array}\right.
$$

where $X, Y$ and $Z$ are Banach spaces, $F: X \rightrightarrows Y$ and $G: X \rightrightarrows Z$ are locally Lipschitz set-valued mappings and $Y^{+} \subset Y$ and $Z^{+} \subset Z$ are closed convex cones. We assume that $\operatorname{dim} Y<+\infty, \operatorname{dim} Z<+\infty$ and that the unit dual ball $\mathbb{B}_{X^{*}}$ is $w^{*}$-sequentially compact.

Our approach consists in using a support function $[1,2,11,12,20]$ together with the schalarization technique proposed by Ciligot-Travain [7] for the study of necessary optimality conditions in vector optimization. In [11], Dien gave a characterization of a set-valued mapping by its support function. The advantage of this characterization is that it allows the theory of generalized derivative of single-valued mappings to be used for set-valued mappings. Fortunately, the 
Lipschitz property of a set-valued mapping is conserved for its support function. Using the above techniques, we establish necessary optimality conditions for the constrained set-valued optimization problem $(P)$. An intermediate set-valued optimization problem is introduced to help us in our investigation. Moreover, as an application, we deduce necessary optimality conditions for a mathematical programming problem.

Our paper is written as follows: in Section 2, we recall some definitions and give some preliminary results. Section 3 is devoted to the optimality conditions. Section 4 discusses an application to a mathematical programming problem.

\section{Preliminaries}

Let $F: X \rightrightarrows Y$ and $G: X \rightrightarrows Z$ be set-valued mappings from $X$ into $Y$ and $Z$. In the sequel, we denote the domain and the graph of $F$ by

$$
\begin{gathered}
\operatorname{dom}(F):=\{x \in X: F(x) \neq \varnothing\}, \\
\operatorname{gr}(F):=\{(x, y) \in X \times Y: y \in F(x)\},
\end{gathered}
$$

respectively. If $V$ is a nonempty subset of $Y$, then

$$
F(V)=\bigcup_{x \in V} F(x) .
$$

A set-valued mapping $F$ is said to be locally Lipschitz at $x \in X$ if there exists a neighborhood $U$ of $x$ such that for some constant $\alpha$ and for all $x_{1}, x_{2} \in U$ we have

$$
F\left(x_{1}\right) \subset F\left(x_{2}\right)+\alpha\left\|x_{1}-x_{2}\right\| \mathbb{B}_{Y}
$$

here $\mathbb{B}_{Y}$ denotes the unit ball of $Y$. The number $\alpha$ is called a Lipschitz constant for $F$ at $x$.

Let $Y^{+} \subset Y$ and $Z^{+} \subset Z$ be pointed closed convex cones such that $\operatorname{Int} Y^{+} \neq$ $\varnothing$. The negative polar cone $Y^{\circ}$ of $Y^{+}$is defined by

$$
Y^{\circ}=\left\{y^{*} \in Y^{*}:\left\langle y^{*}, y\right\rangle \leq 0 \text { for all } y \in Y^{+}\right\},
$$

where $\langle\cdot, \cdot\rangle$ is the dual pair.

Let $A$ be a nonempty subset of $Y$ and $\bar{y} \in A . \bar{y}$ is said to be a weak Pareto minimal point of $A$ with respect to $Y^{+}$if

$$
(A-\bar{y}) \cap\left(-\operatorname{Int} Y^{+}\right)=\varnothing,
$$

here Int denotes the topological interior.

Let $G^{-}\left(-Z^{+}\right):=\left\{x \in X: G(x) \cap-Z^{+} \neq \varnothing\right\}$. A point $(\bar{x}, \bar{y}) \in \operatorname{gr}(F)$ with $x \in G^{-}\left(-Z^{+}\right)$is said to be a weak local Pareto minimal point with respect to $Y^{+}$of the problem $(P)$ if there exists a neighborhood $V$ of $\bar{x}$ such that

$$
F\left(V \cap G^{-}\left(-Z^{+}\right)\right) \subset \bar{y}+Y \backslash\left(-\operatorname{Int} Y^{+}\right) .
$$

Let $y^{*} \in Y$. The function

$$
C_{F}\left(y^{*}, x\right):=\sup _{y \in F(x)}\left\langle y^{*}, y\right\rangle
$$


is called the support function of $F$. It is obvious that $C_{F}\left(y^{*}, \cdot\right)$ is locally Lipschitz in $x$, and $\alpha\left\|y^{*}\right\|$ is a Lipschitz-constant for $C_{F}\left(y^{*}, \cdot\right)$ at $x$ if $\alpha$ is a Lipschitzconstant for $F$ at $x$.

Example 2.1. Let $y^{*} \in\left(Y^{+}\right)^{\circ}$ and $F(x)=f(x)+Y^{+}$, where $f: X \rightarrow Y$ is a locally Lipschitz mapping. In this special case, one has

$$
C_{F}\left(y^{*}, x\right)=\left\langle y^{*}, f(x)\right\rangle \text {. }
$$

Moreover, if $Y^{+}=f\left(f_{1}, f_{2}, \ldots, f_{m}\right)$ and $y^{*}=\left(\lambda_{1}, \lambda_{2}, \ldots, \lambda_{m}\right)$, then

$$
C_{F}\left(y^{*}, x\right)=\sum_{i=1}^{m} \lambda_{i} f(x) .
$$

Assume that the barrier cone of $F(x)$, i.e., the set

$$
Y_{F}^{*}:=\left\{y^{*} \in Y^{*}: \sup _{y \in F(x)}\left\langle y^{*}, y\right\rangle<+\infty\right\}
$$

is closed and does not depend on $x$. This is the case, for example, when $F$ is locally Lipschitz [11].

Suppose that for all $x \in X, F(x)$ is a nonempty, closed and convex set. The distance function of $F$ to zero,

$$
d(y, F(x))=\inf \{\|y-u\|: u \in F(x)\}
$$

is related to the support function of $F$ by

$$
d(y, F(x))=\max _{y^{*} \in Y_{F}^{*} \cap B_{Y^{*}}}\left\langle y^{*}, y\right\rangle-C_{F}\left(y^{*}, x\right) .
$$

If $d(y, F(x))>0$ then there is a unique $y^{*} \in Y_{F}^{*} \cap \mathbb{B}_{Y^{*}}$ satisfying $\left\|y^{*}\right\|=1$ and $d(y, F(x))=\left\langle y^{*}, y\right\rangle-C_{F}\left(y^{*}, x\right)$, see [11] and [20].

For a subset $S$ of $Y$, we consider the function

$$
\Delta_{S}(y)= \begin{cases}d(y, S) & \text { if } y \in Y \backslash S, \\ -d(y, Y \backslash S) & \text { if } y \in S,\end{cases}
$$

where $d(y, S)=\inf \{\|u-y\|: u \in S\}$. This function is introduced in HiriartUrruty [14] (see also [15]), and used by Ciligot-Travain [7], and Amahroq and Taa [3]. Let us recall the following result of [14].

Proposition 2.1 ([14]). Let $S \subset Y$ be a closed convex cone with nonempty interior and $S \neq Y$. The function $\Delta_{S}$ is convex, positively homogeneous, 1 Lipschitzian, decreasing on $Y$ with respect to the order introduced by S. Moreover $(Y \backslash S)=\left\{y \in Y: \Delta_{S}(y)>0\right\}$, Int $(S)=\left\{y \in Y: \Delta_{S}(y)<0\right\}$ and the boundary of $S: \operatorname{bd}(S)=\left\{y \in Y: \Delta_{S}(y)=0\right\}$.

As a direct consequence of Proposition 2.1 one has the following result.

Proposition $2.2([7])$. Let $S \subset \mathbb{R}^{n}$ be a nonempty closed convex cone with nonempty interior. Then for all $y \in \mathbb{R}^{n}, 0 \notin \partial \Delta_{S}(y)$. 
Here, for a locally Lipschitz mapping $f$, the set $\partial f(x)$ denotes the Clarke generalized Jacobian of $f$ at $x$; i.e.,

$$
\partial f(x):=\left\{x^{*} \in X^{*}: \limsup _{u \rightarrow x, t \searrow 0} \frac{f(u+t v)-f(u)}{t} \geq\left\langle x^{*}, v\right\rangle \quad \forall v \in X\right\} .
$$

Recall the following interesting result which is due to Clarke [9]. For more details we refer the interested reader to Proposition 2.3.12 [9].

Proposition $2.3([9])$. Suppose that $\left\{f_{i}\right\}$ is a finite collection of functions $(i=1,2, \ldots, n)$ each of which is Lipschitz near $\bar{x}$. The function $h$ defined by

$$
h(x)=\max \left\{f_{i}(x): i=1,2, \ldots, n\right\}
$$

is easily seen to be Lipschitz near $\bar{x}$ as well. Moreover,

$$
\partial h(\bar{x}) \subset \operatorname{co}\left\{\partial_{f_{i}}(\bar{x}): i \in I(\bar{x})\right\}
$$

where $I(\bar{x}):=\left\{i: f_{i}(\bar{x})=h(\bar{x})\right\}$ and "co" denotes the convex hull.

The next proposition was proved by Dien in 1983; see Proposition 2.2 [11] and $[20]$.

Proposition 2.4. Suppose that $\mathbb{B}_{X^{*}}$ is $w^{*}$-sequentially compact. Then for all $x \in X$ and $y \in Y$,

$$
\partial d(\cdot, F(\cdot))(x, y) \subset \operatorname{co}\left\{\bigcup_{y^{*} \in J(x, y)}-\partial C_{F}\left(y^{*}, \cdot\right)(x) \times\left\{y^{*}\right\}\right\},
$$

where $J(x, y)=\left\{y^{*} \in Y_{F}^{*}:\left\|y^{*}\right\| \leq 1, d(y, F(x))=\left\langle y^{*}, y\right\rangle-C_{F}\left(y^{*}, x\right)\right\}$.

If, in addition, $d(y, F(x))>0$, then $J(x)$ consists of only one single element $y^{*}$ with $\left\|y^{*}\right\|=1$ and the symbol "co" can be deleted.

In what follows, we suppose that $\operatorname{dim} Y<+\infty$, $\operatorname{dim} Z<+\infty$ and that $\mathbb{B}_{X^{*}}$ is $w^{*}$-sequentially compact. Moreover, the set-valued mappings $F$ and $G$ are assumed to have the following properties:

i. The set-valued mapping $\left(y^{*}, x\right) \rightarrow \partial C_{F}\left(y^{*}, \cdot\right)(x)$ is upper semicontinuous when $X^{*}, Y^{*}$ are endowed with the weak-star topology and $X$ with the strong topology, that is, if $x_{n}^{*} \in \partial C_{F}\left(y_{n}^{*}, \cdot\right)\left(x_{n}\right)$ where $x_{n}^{*} \stackrel{w^{*}}{\rightarrow} x^{*}$ in $X^{*}, y_{n}^{*} \stackrel{w^{*}}{\rightarrow} y^{*}$ in $Y^{*}$ and $x_{n} \rightarrow x$ in $X$, then $x^{*} \in \partial C_{F}\left(y^{*}, \cdot\right)(x)$.

ii. The set-valued mapping $\left(z^{*}, x\right) \rightarrow \partial C_{G}\left(z^{*}, \cdot\right)(x)$ is upper semicontinuous when $X^{*}, Z^{*}$ are endowed with the weak-star topology and $X$ with the strong topology, that is, if $x_{n}^{*} \in \partial C_{G}\left(z_{n}^{*}, \cdot\right)\left(x_{n}\right)$ where $x_{n}^{*} \stackrel{w^{*}}{\rightarrow} x^{*}$ in $X^{*}, z_{n}^{*} \stackrel{w^{*}}{\rightarrow} z^{*}$ in $Z^{*}$ and $x_{n} \rightarrow x$ in $X$, then $x^{*} \in \partial C_{G}\left(z^{*}, \cdot\right)(x)$.

Remark 2.1. 1. The above property was introduced by Dien (see [11, 12]). He calls it the Cl-property.

2. In some cases, the Cl-property can be established without difficulty. See the following example.

Example 2.2. Let $y^{*} \in\left(Y^{+}\right)^{\circ}$ and $F(x)=f(x)+Y^{+}$, where $f: X \rightarrow Y$ is a locally Lipschitz mapping. 
Suppose that $x_{n}^{*} \in \partial C_{F}\left(y_{n}^{*}, \cdot\right)\left(x_{n}\right)$, where $x_{n}^{*} \stackrel{w^{*}}{\rightarrow} x^{*}$ in $X^{*}, y_{n}^{*} \rightarrow y^{*}$ in $Y^{*}$ and $x_{n} \rightarrow x$ in $X$. Remarking that $C_{F}\left(y_{n}^{*}, x_{n}\right)=\left\langle y_{n}^{*}, f\left(x_{n}\right)\right\rangle$ and that $x_{n}^{*} \in$ $\partial\left\langle y_{n}^{*}, f\right\rangle\left(x_{n}\right)$, we deduce that $x^{*} \in \partial\left\langle y^{*}, f\right\rangle(x)$.

\section{Optimality Conditions}

Consider the unconstrained multiobjective optimization problem

$$
\left(P_{1}\right):\left\{\begin{array}{c}
\min F(x), \\
\text { subject to } x \in X,
\end{array}\right.
$$

where $F$ is a set-valued mapping defined from $X$ into $Y$. Theorem 1 gives necessary optimality conditions for the optimization problem $\left(P_{1}\right)$.

Theorem 1. Suppose that $F$ is locally Lipschitz at $\bar{x}$. If $(\bar{x}, \bar{y}) \in \operatorname{gr}(F)$ is a weak local Pareto minimal point with respect to $Y^{+}$of the problem $\left(P_{1}\right)$, then there exists $y^{*} \in\left(Y^{+}\right)^{\circ} \backslash\{0\}$ such that

$$
\left\{\begin{array}{l}
0 \in \partial C_{F}\left(y^{*}, \cdot\right)(\bar{x}) \\
C_{F}\left(y^{*}, \bar{x}\right)=\left\langle y^{*}, \bar{y}\right\rangle
\end{array}\right.
$$

Proof. By assumption, there exists a neighborhood $V$ of $\bar{x} \in X$ such that

$$
F(x) \subset \bar{y}+Y \backslash\left(-\operatorname{Int} Y^{+}\right) \text {for all } x \in V \text {. }
$$

That is for all $x \in V$ and $y \in F(x)$ one has

$$
y-\bar{y} \in Y \backslash\left(-\operatorname{Int} Y^{+}\right) \text {, }
$$

hence by Proposition 2.1, $\Delta_{-\operatorname{Int} Y^{+}}(y-\bar{y}) \geq 0$.

Since $\Delta_{-\operatorname{Int} Y^{+}}(0)=0$, it follows that $(\bar{x}, \bar{y})$ solves locally the problem

$$
\text { Minimize } \Delta_{-\operatorname{Int} Y^{+}}(y-\bar{y}) \text { subject to } y \in F(x) \text {. }
$$

Let $u=(x, y)$ and $\bar{u}=(\bar{x}, \bar{y})$.

Let $\Psi_{1}(u):=\Delta_{-\operatorname{Int} Y^{+}}(y-\bar{y})+\frac{1}{n}, \Psi_{2}(u):=d(y, F(x))$ and $h_{n}(u):=$ $\max \left(\Psi_{1}(u), \Psi_{2}(u)\right)$; we have $h_{n}(\bar{u}) \leq \frac{1}{n}+\inf _{u \in X \times Y} h_{n}(u)$.

By Ekeland's variational principle [13] there exists $u_{n} \in X$ such that

$$
\left\{\begin{array}{c}
\left\|u_{n}-\bar{u}\right\| \leq \frac{1}{\sqrt{n}}, \\
h_{n}\left(u_{n}\right) \leq h_{n}(u)+\frac{1}{\sqrt{n}}\left\|u-u_{n}\right\| \quad \text { for all } u \in X \times Y .
\end{array}\right.
$$

Hence $u_{n}$ is a minimum of $h_{n}(u)+\frac{1}{\sqrt{n}}\left\|u-u_{n}\right\|$ and we get

$$
0 \in \partial h_{n}\left(u_{n}\right)+\frac{1}{\sqrt{n}} \mathbb{B}_{X^{*} \times Y^{*}}
$$

In view of Proposition 2.3 it follows that $\partial h_{n} \subset \operatorname{co}\left\{\partial \Psi_{i}: i \in I\left(u_{n}\right)\right\}$, where

$$
I\left(u_{n}\right):=\left\{i: h_{n}\left(u_{n}\right)=\Psi_{i}\left(u_{n}\right)\right\} .
$$

Consequently, there exists $\lambda_{n} \in[0,1]$ such that

$$
0 \in\left(1-\lambda_{n}\right) \partial \Psi_{1}\left(u_{n}\right)+\lambda_{n} \partial \Psi_{2}\left(u_{n}\right)+\frac{1}{\sqrt{n}} \mathbb{B}_{X^{*} \times Y^{*}}
$$


where $\lambda_{n}=1$ if $\Psi_{1}\left(u_{n}\right)<\Psi_{2}\left(u_{n}\right), \lambda_{n}=0$ if $\Psi_{2}\left(u_{n}\right)<\Psi_{1}\left(u_{n}\right)$ and $0<\lambda_{n}<1$ if $\Psi_{1}\left(u_{n}\right)=\Psi_{2}\left(u_{n}\right)$.

- $\max \left(\Psi_{1}\left(u_{n}\right), \Psi_{2}\left(u_{n}\right)\right)>0$ for $n$ large enough, otherwise

$$
\left\{\begin{array}{l}
d\left(y_{n}, F\left(x_{n}\right)\right)=0 \\
\Delta_{-\operatorname{Int}\left(Y^{+}\right)}\left(y_{n}-\bar{y}\right)+\frac{1}{n} \leq 0 .
\end{array}\right.
$$

Therefore $y_{n} \in F\left(x_{n}\right)$ and $\Delta_{-\operatorname{Int} Y^{+}}(y-\bar{y}) \leq-\frac{1}{n}$. Since $\bar{u}$ is a weak local Pareto minimal point with respect to $Y^{+}$of the problem $\left(P_{1}\right)$, one has

$$
F\left(x_{n}\right) \subset \bar{y}+Y /-\left(\operatorname{Int} Y^{+}\right) .
$$

Consequently, $\Delta_{-\operatorname{Int} Y^{+}}\left(y_{n}-\bar{y}\right) \geq 0$, which is a contradiction.

- $\Psi_{2}\left(u_{n}\right)=d\left(y_{n}, F\left(x_{n}\right)\right)>0$ for $n$ large enough. Otherwise, $\Psi_{2}\left(u_{n}\right)=0$. Since $\max \left(\Psi_{1}\left(u_{n}\right), \Psi_{2}\left(u_{n}\right)\right)>0$, we deduce that $\Psi_{2}\left(u_{n}\right)<\Psi_{1}\left(u_{n}\right)$. Consequently, from (1),

$$
\lambda_{n}=0 \text { and } 0 \in \partial \Psi_{1}\left(u_{n}\right)+\frac{1}{\sqrt{n}} \mathbb{B}_{Y^{*}} .
$$

Since $\partial \Delta_{-\operatorname{Int} Y^{+}}($.$) is closed, one gets 0 \in \partial \Delta_{- \text {Int } Y^{+}}(0)$. It is a contradiction to Proposition 2.2.

Using Proposition 2.4, there exist $y_{n}^{*} \in Y_{F}^{*} \cap \mathbb{S}_{Y^{*}}$ and a real number $\left.\lambda_{n} \in\right] 0,1[$ such that

$$
\left\{\begin{array}{l}
0 \in-\lambda_{n} \partial C_{F}\left(y_{n}^{*}, .\right)\left(x_{n}\right)+\frac{1}{\sqrt{n}} \mathbb{B}_{X^{*}}, \\
0 \in\left(1-\lambda_{n}\right) \partial \Delta_{-\operatorname{Int} Y^{+}}\left(y_{n}-\bar{y}\right)+\lambda_{n} y_{n}^{*}+\frac{1}{\sqrt{n}} \mathbb{B}_{Y^{*}}
\end{array}\right.
$$

Hence there is $m_{n}^{*} \in \partial \Delta_{-\operatorname{Int} Y^{+}}\left(y_{n}-\bar{y}\right)$ such that

$$
\left\{\begin{array}{l}
0 \in-\lambda_{n} \partial C_{F}\left(y_{n}^{*}, .\right)\left(x_{n}\right)+\frac{1}{\sqrt{n}} \mathbb{B}_{X^{*}}, \\
0 \in\left(1-\lambda_{n}\right) m_{n}^{*}+\lambda_{n} y_{n}^{*}+\frac{1}{\sqrt{n}} \mathbb{B}_{Y^{*}}
\end{array}\right.
$$

Since $\operatorname{dim} Y$ is finite, taking a subsequence if necessary, we can assume that

$$
\left\{\begin{array}{l}
\left(\lambda_{n}\right) \rightarrow \lambda \in[0,1], \\
\left(y_{n}^{*}\right) \rightarrow \widetilde{y}^{*} \in Y_{F}^{*} \cap \mathbb{S}_{Y^{*}},
\end{array}\right.
$$

when $n \rightarrow+\infty$.

- Since $\partial \Delta_{-\operatorname{Int} Y^{+}}($.$) is upper semicontinuous and closed, due to Proposi-$ tion 2.2, there exist $m^{*} \in \partial \Delta_{-\operatorname{Int} Y^{+}}(0), m^{*} \neq 0_{Y^{*}}$ and a subsequence $\left(m_{\varphi(n)}^{*}\right)$ of $\left(m_{n}^{*}\right)$ such that $m_{\varphi(n)}^{*} \rightarrow m^{*}$. Moreover, since $\Delta_{-\operatorname{Int} Y^{+}}($.$) is a$ convex function and $\Delta_{-\operatorname{int} Y^{+}}(0)=0$, we have for all $y \in Y$

$$
\Delta_{-\operatorname{int} Y^{+}}(y) \geq\left\langle m^{*}, y\right\rangle,
$$

and hence for all $y \in-Y^{+}$

$$
\left\langle m^{*}, y\right\rangle \leq \Delta_{-\operatorname{int} Y^{+}}(y)=-d\left(y, Y \backslash-\operatorname{Int} Y^{+}\right) \leq 0 .
$$


Therefore $m^{*} \in\left(-Y^{+}\right)^{\circ}$.

- One the one hand, $\lambda>0$. Otherwise, by $(2), m^{*}=0$, which is a contradiction.

On the other hand, $\lambda<1$. Otherwise, by $(2), \widetilde{y}^{*}=0$, which is a contradiction.

Assuming that $\alpha$ is a Lipschitz constant for $F$ at $\bar{x}$,

$$
C_{F}\left(y_{n}^{*}, \bar{x}\right)-\left\langle y_{n}^{*}, \bar{y}\right\rangle \leq C_{F}\left(y_{n}^{*}, x_{n}\right)-\left\langle y_{n}^{*}, \bar{y}\right\rangle+\alpha\left\|y_{n}^{*}\right\|\left\|x_{n}-\bar{x}\right\| .
$$

Letting $n \rightarrow+\infty$, we get

$C_{F}\left(\widetilde{y}^{*}, \bar{x}\right)-\left\langle\widetilde{y}^{*}, \bar{y}\right\rangle \leq \liminf _{n \rightarrow+\infty}\left(C_{F}\left(y_{n}^{*}, x_{n}\right)-\left\langle y_{n}^{*}, y_{n}\right\rangle\right) \leq \lim _{n \rightarrow+\infty}-d\left(y_{n}, F\left(x_{n}\right)\right)=0$.

Since $\bar{y} \in F(\bar{x})$, we have $C_{F}\left(\widetilde{y}^{*}, \bar{x}\right)=\left\langle\widetilde{y}^{*}, \bar{y}\right\rangle$. Finally,

$$
\left\{\begin{array}{l}
0 \in \partial C_{F}\left(y^{*}, .\right)(\bar{x}) \\
C_{F}\left(y^{*}, \bar{x}\right)=\left\langle y^{*}, \bar{y}\right\rangle
\end{array}\right.
$$

with $y^{*}=\frac{1}{\lambda} \widetilde{y}^{*}$.

In order to give necessary optimality conditions for the multiobjective optimization problem $(P)$, we introduce the intermediate problem $\left(P_{2}\right)$.

In the sequel $(F, G)$ will be the multifunction from $X$ into $Y \times Z$ defined by $(F, G)(x)=(F(x), G(x))=F(x) \times G(x)$ for all $x \in \operatorname{dom}(F) \cap \operatorname{dom}(G)$.

Let $\bar{z} \in G(\bar{x}) \cap\left(-Z^{+}\right)$and consider the next problem $\left(P_{2}\right)$ with respect to $Y^{+} \times\left(Z^{+}+\bar{z}\right)$

$$
\begin{aligned}
& \left(P_{2}\right): \quad \text { Minimize }(F, G)(x) \\
& \quad \text { Subject to } x \in X
\end{aligned}
$$

Proposition 3.1 compares the set of all Pareto minimal points of $(P)$ and the set of all Pareto minimal points of $\left(P_{2}\right)$. This result is proved by Amahroq and Taa in [4]. For the convenience of the reader, the proof is given below.

Proposition 3.1. Suppose that $F$ and $G$ are locally Lipschitz at $\bar{x}$. Let $(\bar{x}, \bar{y}) \in$ $\operatorname{gr}(F)$ with $G(\bar{x}) \cap\left(-Z^{+}\right) \neq \varnothing$. If $(\bar{x}, \bar{y})$ is a local weak Pareto minimal point of $(P)$ with respect to $Y^{+}$, then for all $\bar{z} \in G(\bar{x}) \cap\left(-Z^{+}\right),(\bar{x}, \bar{y}, \bar{z})$ is a local weak Pareto minimal point of $\left(P_{2}\right)$ with respect to $Y^{+} \times\left(Z^{+}+\bar{z}\right)$.

Proof. Suppose the contrary. There exists $\bar{z} \in G(\bar{x}) \cap\left(-Z^{+}\right)$such that $(\bar{x}, \bar{y}, \bar{z})$ is not a local weak Pareto minimal point of $\left(P_{2}\right)$. One can find sequences $\left(x_{n}\right) \rightarrow$ $\bar{x},\left(y_{n}\right) \rightarrow \bar{y}$ and $\left(z_{n}\right) \subset Z$ such that for all $n \in \mathbb{N}$

$$
y_{n} \in F\left(x_{n}\right), z_{n} \in G\left(x_{n}\right)
$$

and

Thus

$$
(\bar{x}, \bar{y})-\left(y_{n}, z_{n}\right) \in \operatorname{Int}\left[Y^{+} \times\left(Z^{+}+\bar{z}\right)\right]
$$

$$
y_{n} \in F\left(x_{n}\right), z_{n} \in G\left(x_{n}\right) \cap\left(-Z^{+}\right) \text {and } \bar{y}-y_{n} \in \operatorname{Int} Y^{+} .
$$

A contradiction, since $(\bar{x}, \bar{y})$ is a local weak Pareto minimal point of $(P)$ with respect to $Y^{+}$. 
As a consequence of Theorem 1 and Proposition 3.1 one has the following result.

Corollary 1. Let $(\bar{x}, \bar{y}) \in \operatorname{gr}(F)$ with $G(\bar{x}) \cap\left(-Z^{+}\right) \neq \varnothing$. If $(\bar{x}, \bar{y})$ is a local weak Pareto minimal point of $(P)$ with respect to $Y^{+}$, then for all $\bar{z} \in$ $G(\bar{x}) \cap\left(-Z^{+}\right)$there exist $y^{*} \in\left(Y^{+}\right)^{\circ}$ and $z^{*} \in\left(Y^{+}\right)^{\circ},\left(y^{*}, z^{*}\right) \neq\left(0_{Y^{*}}, 0_{Z^{*}}\right)$ such that

$$
\left\{\begin{array}{l}
0 \in \partial C_{F}\left(y^{*}, \cdot\right)(\bar{x})+\partial C_{G}\left(z^{*}, \cdot\right)(\bar{x}), \\
C_{F}\left(y^{*}, \bar{x}\right)+C_{G}\left(z^{*}, \bar{x}\right)=\left\langle y^{*}, \bar{y}\right\rangle+\left\langle z^{*}, \bar{z}\right\rangle .
\end{array}\right.
$$

Remark 3.1. Using a regularity condition, one can obtain $y^{*} \neq 0_{Y^{*}}$. Such a condition is given by Dien in [12]. The proof will be similar to that of Theorem 1.

As a special case, take the following optimization problem

$$
\begin{gathered}
\text { Min } f(x) \\
\left(P_{3}\right): \quad \text { Subject to }-g(x) \in Z^{+}
\end{gathered}
$$

where $f: X \rightarrow Y$ and $g: X \rightarrow Z$ are locally Lipschitz mappings.

Corollary 2. Suppose that $(\bar{x}, f(x))$ is a local weak Pareto minimal point of $\left(P_{3}\right)$ with respect to $Y^{+}$. Then there exist $y^{*} \in\left(Y^{+}\right)^{\circ}$ and $z^{*} \in\left(Y^{+}\right)^{\circ},\left(y^{*}, z^{*}\right) \neq$ $\left(0_{Y^{*}}, 0_{Z^{*}}\right)$ such that

$$
\left\{\begin{array}{c}
0 \in \partial\left\langle y^{*}, f\right\rangle(\bar{x})+\partial\left\langle z^{*}, g\right\rangle(\bar{x}), \\
\left\langle z^{*}, g\right\rangle=0
\end{array}\right.
$$

By the following example we illustrate the usefulness of the necessary conditions in Corollary 1.

Example 3.1. Let $f=\left(f_{1}, \ldots, f_{n}\right), g=\left(g_{1}, \ldots, g_{n}\right): X \rightarrow \mathbb{R}^{n}$ be given mappings. We consider the set-valued mappings $F$ and $G: X \rightrightarrows \mathbb{R}^{n}$ with

$$
\left\{\begin{array}{l}
F(x):=\left\{y \in \mathbb{R}^{n}: y-f(x) \in \mathbb{R}_{+}^{n}\right\}, \\
G(x):=\left\{z \in \mathbb{R}: z-g(x) \in \mathbb{R}_{+}^{n}\right\} .
\end{array}\right.
$$

Under these assumptions, we investigate the optimization problem

$$
\left(P^{\diamond}\right):\left\{\begin{array}{c}
\min F(x) \\
\text { subject to } G(x) \cap-\mathbb{R}_{+}^{n} \neq \varnothing .
\end{array}\right.
$$

This is a special case of the general type $(P)$. In this example, the values of the objective may vary between the values of two known functions.

Next, assume that $(\bar{x}, f(\bar{x}))$ is a weak local Pareto minimal point of $\left(P^{\diamond}\right)$ and that $f$ and $g$ are locally Lipschitz at $\bar{x} \in\left\{x \in X: G(x) \cap-\mathbb{R}_{+}^{n} \neq \varnothing\right\}$. Consequently, $F$ and $G$ are locally Lipschitz at $\bar{x}$. Then there exist vectors $\lambda=\left(\lambda_{1}, \lambda_{2}, \ldots, \lambda_{m}\right) \in \mathbb{R}_{+}^{m}$ and $\mu=\left(\mu_{1}, \mu_{2}, \ldots, \mu_{k}\right) \in \mathbb{R}^{k},(\lambda, \mu) \neq(0,0)$ such that

$$
\left\{\begin{array}{c}
0 \in \sum_{i=1}^{m} \lambda_{i} \partial f_{i}(\bar{x})+\sum_{j=1}^{k} \mu_{j} \partial g_{j}(\bar{x}), \\
\lambda_{i} g_{i}(\bar{x})=0, \quad i=1,2, \ldots, m .
\end{array}\right.
$$




\section{Applichtion}

In this section we are concerned with the mathematical programming problem

$$
\begin{aligned}
& \min f(x), \\
\left(P^{*}\right): & \begin{cases}g_{i}(x) \leq 0, & i=1,2, \ldots, m, \\
h_{j}(x)=0, & j=1,2, \ldots, k,\end{cases}
\end{aligned}
$$

where $f, g_{i}$, and $h_{j}$ are locally Lipschitz at $\bar{x}$.

Setting $C:=\left\{x: g_{i}(x) \leq 0, h_{j}(x)=0\right.$ for all $\left.i, j\right\}, g(x)=\left(g_{1}(x), g_{2}(x)\right.$, $\left.\ldots, g_{m}(x)\right)$ and $h(x)=\left(h_{1}(x), h_{2}(x), \ldots, h_{k}(x)\right)$, problem $\left(P^{*}\right)$ is reduced to problem $(P)$ when the set-valued mapping $G$ from $X$ into $Z=\mathbb{R}^{m} \times \mathbb{R}^{k}$ is defined by

$$
G(x):=(g(x), h(x))+\mathbb{R}_{+}^{m} \times\left\{0_{\mathbb{R}^{k}}\right\} ;
$$

here $\mathbb{R}_{+}^{m}$ is the nonnegative orthant of $\mathbb{R}^{m}$.

Obviously in that case, $Z^{+}=\mathbb{R}_{+}^{m+k}$ and for any $z^{*}=(\lambda, \mu) \in Z^{+}$we have

$$
C_{G}\left(z^{*}, x\right)=\langle\lambda, g(x)\rangle+\langle\mu, h(x)\rangle .
$$

Take $\bar{x} \in C, \bar{z}=0 \in G(\bar{x}) \cap Z^{+}$and $z^{*}=(\lambda, \mu) \in \mathbb{R}_{+}^{m+k}$; it can be verified that $C_{G}\left(z^{*}, \bar{x}\right)=0$ if and only if $\langle\lambda, g(\bar{x})\rangle=0$.

In conclusion, we deduce from the Theorem 1 the following necessary condition for problem $\left(P^{*}\right)$.

Theorem 2. Let $\bar{x}$ be a solution of $\left(P^{*}\right)$. Then there exist vectors $\lambda=$ $\left(\lambda_{0}, \lambda_{1}, \lambda_{2}, \ldots, \lambda_{m}\right) \in \mathbb{R}_{+}^{m+1}$ and $\mu=\left(\mu_{1}, \mu_{2}, \ldots, \mu_{k}\right) \in \mathbb{R}^{k},(\lambda, \mu) \neq(0,0)$ such that

$$
\left\{\begin{array}{c}
0 \in \lambda_{0} \partial f(\bar{x})+\sum_{i=1}^{m} \lambda_{i} \partial g_{i}(\bar{x})+\sum_{j=1}^{k} \mu_{j} \partial h_{j}(\bar{x}), \\
\lambda_{i} g_{i}(\bar{x})=0, \quad i=1,2, \ldots, m .
\end{array}\right.
$$

Remark 4.1. In this special case, in order to get $\lambda_{0} \neq 0$, it suffices to have the vectors $\nabla g_{i}(\bar{x}), i \in q(\bar{x}), \nabla h_{j}(\bar{x}), j=1, \ldots, k$, linearly independent.

\section{ACKNOWLEDGMENT}

I extend my sincere acknowledgements to the referees for his/her insightful remarks and to Pr. T. Amahroq for his suggestions which improved the original version of this work.

\section{REFERENCES}

1. T. Amahroq and N. Gadhi, Second order optimality conditions for the extremal problem under inclusion constraints. J. Math. Anal. Appl. 285(2003), No. 1, 74-85.

2. T. Amahroq, N. Gadhi, and H. Riahi, Epi-differentiability and optimality conditions for an extremal problem under inclusion constraints. JIPAM. J. Inequal. Pure Appl. Math. 4(2003), No. 2, Article 41, 11 pp. (electronic).

3. T. Amahroq and A. TAA, On Lagrange-Kuhn-Tucker multipliers for multiobjective optimization problems. Optimization 41(1997), No. 2, 159-172. 
4. T. Amahroq and A. TAA, Optimality conditions for multiobjective problems with paraconvex data. Studia Math. (to appear).

5. J. M. Borwein, Multivalued convexity and optimization: a unified approach to inequality and equality constraints. Math. Programming 13(1977), No. 2, 183-199.

6. J. M. Borwein, A Lagrange multiplier theorem and a sandwich theorem for convex relations. Math. Scand. 48(1981), No. 2, 189-204.

7. M. C. Travain, On Lagrange-Kuhn-Tucker multipliers for Pareto optimization problems. Numer. Funct. Anal. Optim. 15(1994), No. 5-6, 689-693.

8. F. H. Clarke, Necessary conditions for a general control problem. Calculus of variations and control theory (Proc. Sympos., Math. Res. Center, Univ. Wisconsin, Madison, Wis., 1975; dedicated to Laurence Chisholm Young on the occasion of his 70th birthday), 257278. Publ. Math. Res. Center Univ. Wisconsin, No. 36, Academic Press, New York, 1976.

9. F. H. Clarke, Optimization and nonsmooth analysis. Canadian Mathematical Society Series of Monographs and Advanced Texts. A Wiley-Interscience Publication. John Wiley \& Sons, Inc., New York, 1983.

10. H. W. Corley, Optimality conditions for maximizations of set-valued functions. $J$. Optim. Theory Appl. 58(1988), No. 1, 1-10.

11. P. H. Dien, Locally Lipschitzian set-valued maps and generalized extremal problems with inclusion constraints. Acta Math. Vietnam. 8(1983), No. 1, 109-122 (1984).

12. P. H. Dien, On the regularity condition for the extremal problem under locally Lipschitz inclusion constraints. Appl. Math. Optim. 13(1985), No. 2, 151-161.

13. I. Ekeland, On the variational principle. J. Math. Anal. Appl. 47(1974), 324-353.

14. J. B. Hiriart-Urruty, Tangent cones, generalized gradients and mathematical programming in Banach spaces. Math. Oper. Res. 4(1979), No. 1, 79-97.

15. J. B. Hiriart-Urruty and C. Lemaréchal, Convex analysis and minimization algorithms. I. Fundamentals. Grundlehren der Mathematischen Wissenschaften [Fundamental Principles of Mathematical Sciences], 305. Springer-Verlag, Berlin, 1993.

16. J. Jahn And R. RAuH, Contingent epiderivatives and set-valued optimization. Math. Methods Oper. Res. 46(1997), No. 2, 193-211.

17. Z. LI, A theorem of the alternative and its application to the optimization of set-valued maps. J. Optim. Theory Appl. 100(1999), No. 2, 365-375.

18. D. T. LuC, Contingent derivatives of set-valued maps and applications to vector optimization. Math. Programming 50(1991), No. 1 (Ser. A), 99-111.

19. D. T. Luc and C. Malivert, Invex optimisation problems. Bull. Austral. Math. Soc. 46(1992), No. 1, 47-66.

20. L. Thibault, On subdifferentials of optimal value functions. SIAM J. Control Optim. 29(1991), No. 5, 1019-1036.

(Received 1.04.2003)

Author's address:

Faculty of Sciences Dhar El Mehrez

Sidi Mohamed Ben Abdellah University

Fes, Morocco

E-Mail:n.gadhi@ucam.ac.ma 